\title{
Analysis of single and multiple on-chip antenna for intra-chip wireless communication with four antenna transceiver model
}

\author{
Deepa N P* and K L Sudha \\ Dayananda Sagar College of Engineering, Visvesvaraya Technological University, Bengaluru, Karnataka
}

Received: 22-May-2021; Revised: 15-July-2021; Accepted: 19-July-2021

(C)2021 Deepa N P and K L Sudha. This is an open access article distributed under the Creative Commons Attribution (CC BY) License, which permits unrestricted use, distribution, and reproduction in any medium, provided the original work is properly cited.

\begin{abstract}
The Radio Frequency $(R F)$ wireless interconnect is one way to improve the performance of multi-core chips that is limited by high power dissipation in the form of heat in conventional metal wired connections. This paper examined the suitability of various existing on-chip antennas, as well as the performance of four such antennas located symmetrically at the four edges of the chip substrate for short-range millimeter-wave intra-chip communication. In order to simulate the scenario of RF transceiver, millimeter-wave antenna on-chip models were developed using the CST MWS software tool. The simulation is carried out for mm-wave range of 10-75 $\mathrm{GHz}$. In the first scenario, zig-zag antenna, planar triangular monopole antenna, bowtie antenna and rectangular meander antenna were designed for the specified frequency range. Different parameters were observed and compared to the same. The approximate resonating frequency and return loss (S11) are $75 \mathrm{GHz}$ and $17 \mathrm{~dB}$, respectively for the meander and zig-zag antenna. In case of bowtie, it was $-46 \mathrm{~dB}$, which is acceptable, but $-6.81 \mathrm{~dB}$ for the triangular monopole which is insignificant. In the second scenario, multiple antenna transceiver models were designed using the combination of these antennas. Meander antenna was found to perform better, with a lower reflection coefficient and a good Voltage Standing Wave Ratio (VSWR). The transmission coefficient of the side antenna is 4 to 16 dB higher than the oppositely placed antenna in the four-antenna model where antennas are kept at the edge centre. Most of the designs have a VSWR between 1 and 2 at their resonant frequency which is satisfactory.

\section{Keywords}

On-chip antenna, WiNoC, Meander antenna, Zig-zag antenna, Short-range communication, Intra chip communication.
\end{abstract}

\section{Introduction}

A communication channel can be wire-line or wireless. A channel can be used for unicast, multicast or broadcast systems. Wireless communication refers to the electromagnetic transfer of data between two or more points. Communication over radio waves requires an antenna [1-3]. An interconnect connects two electronic devices or networks. Some of the nonwired technologies are RF wireless interconnects, optical interconnects, 3D chips, Alternating Current (AC) coupling, etc. Generally, interconnects are low frequency metal wired connections. An unlicensed spectrum in the range of $60 \mathrm{GHz}$ frequency has gained much more attention in the last few years due to increased demand for very high-speed multimedia wireless short-range communications [5-8].

*Author for correspondence

874
The $60 \mathrm{GHz}$ systems have become feasible for mass production with the development of silicon technology and have paved the way for the integration of Radio Frequency (RF) components with analog and digital circuitry on a single chip, like System on Chip (SoC) with a much more compact size.

Modern communication systems require the transmission of very high bit rates, due to which the traditional metal wiring interconnects technology brings with it serious problems such as signal integrity, increased latency, and power dissipation, which is a major hurdle to improve the efficiency of the Very Large-Scale Integration (VLSI) system [9-14]. Therefore, future Integrated Circuits (IC) require nonwired interconnections. In order to make the interconnects wireless, the antenna plays a major role. In addition, it is necessary to consider the placement of the antenna and the type of antenna in such a scenario. This study compares the behaviour of four antenna models placed in the edge centers. RF transceiver with on-chip antenna technique has been increasingly researched in the past years. On-chip 
antennas are primarily used as wireless interconnects for intra-chip/inter-chip communications.

This research focuses on constructing various millimeter-wave on-chip antennas of different geometries using CST MWS software. The frequency range covered from 10-70 GHz. The performance parameters covered in this study were return loss (S11), Voltage Standing Wave Ratio (VSWR), polar plot, directivity and radiation pattern of a various simulated single antenna. To study the channel behaviour between the transceiver antennas, four antenna transceiver model using the single antennas designed were constructed and analysed using the Sparameters. Based on the investigation of the past work, four antennas were chosen which are zig-zag antenna, planar triangular monopole antenna, bowtie antenna and rectangular meander antenna. The antennas were remodelled, if the deviation from expected results were obtained and appropriate results were recorded.

In 3D ICs, circuits are divided into smaller parts and placed in layers as stacks, avoiding the long wires, but this method has still not gone commercial because of the complicated etching and fabrication process involved in this method. Heat removal is a challenging task with respect to the increase in power density in 3D chips. Optical interconnects offer transmission at the speed of light without the challenges of frequency, dispersion and losses associated with the conventional electrical wiring. The transformation of the electrical signal to optical signal requires the use of special devices such as optical modulator and demodulator for which special packaging process and technology are required. All these requirements lead to a costly and complex fabrication process. The interconnect system based on mainly capacitive and inductive coupling, also known as AC Coupling doesn't require any extra manufacturing and packaging process as compared to the $3 \mathrm{D}$ chips and optical interconnect technology. However, there is a constraint on the implementation of this technology due to the inability to support larger distances and the requirement of accurate alignment for good performance. Radio Frequency (RF) transceiver based wireless communication avoids the problem of complex packaging and the technology involved in using optical signals and the constraints of distance in case of AC coupling interconnects.

The organization of the research paper is as follows. In Section 2, related work was discussed. The construction, working of single and 4 antenna models were discussed in Section 3 followed by results and performance of channel models in Section 4. Finally, conclusions and future scope were drawn in Section 5.

\section{Related work}

The shrinking of antennas and transceivers supports the improvement of Wireless-Networks-on-Chip (WiNoC), in which chip-scale communication is employed to surge the computing performance of multi-core architectures. Traditional wired interconnects have many limitations and to overcome these limitations wireless interconnect systems have been employed. Wireless interconnection systems recognize intra-chip interconnection at light speed through electromagnetic waves [1]. The electromagnetic modeling and designing of on-chip dipole antenna were presented in [2]. The obtained results prove that the practicability of this approach and opens opportunities for antenna integration on a chip and the fabrication of totally integrated singlechip wireless systems. In reference [3] the requirements and performance of some of the proposed non-wired interconnect technologies such as optical interconnects, Through-Silicon-Via (TSVs), AC coupling and RF transceiver based wireless interconnects are discussed and compared. The Radio Frequency Wireless Interconnect (RFWI) method was further analysed by modelling in the Simulink environment, and efficient communication between transceivers was obtained. The results point out the possible reliability and feasibility of the intra-chip and inter-chip communication system by using an integrated RF transceiver and antenna to replace the existing interconnecting metal wiring. In the succeeding research carried out by Chang et al. [4], a metallic zig-zag antenna was demonstrated to provide the best power gain for the smallest area overhead. A 3 -dB bandwidth of $16 \mathrm{GHz}$ having a center frequency of $57.5 \mathrm{GHz}$ was obtained by using the chip model. A triangular monopole antenna of high gain and low area using a standard Complementary Metal Oxide Semiconductor (CMOS) process was discussed in [5]. Optimization over a modified rectangular Artificial Magnetic Conductor (AMC) which acts as a guard between the Antenna-on-Chip (AOC) and the lossy CMOS substrate was also discussed in [5]. The design of an on-chip Planar Log-Periodic Antenna (PLPA) [6] was proposed in 2014. The PLPA antenna was designed to enhance the signal transmission features of wireless interconnections, then a two-port antenna network was also simulated for $60 \mathrm{GHz}$ in HighFrequency Structure Simulator (HFSS) tool and the radiation pattern and the scattering matrix parameters were achieved. In the year 2016, a simplified Wireless Inter/Intra-Chip (WIIC) communication system [7] 
prototype was studied using an innovative metamaterial Printed Circuit Board (PCB) absorber propagation channel. Simulation modeling that predicts Bit Error Rate (BER) in High-Speed Interconnect (HIS) systems are presented in reference [8]. The solution offers a practical method for analyzing the system using a novel 3D bathtub curve [8]. The on-chip wireless channel and the effect of antenna positioning and directionality on channel characteristics are described in recent research studies [9]. Analysis of the intra-chip environment reveals that it differs greatly from free space and creates smallscale fading effects from various propagation paths from both chip layers and chip edges. Elaiyabharathi et al. [10] presented a design of the meander line antenna for wireless application for the operating frequency of $3.4 \mathrm{GHz}$ like Worldwide Interoperability for Microwave Access (WiMAX). The antenna performance was improved based on the reflection coefficient of the antenna, radiation pattern, VSWR, and return loss. The return loss was calculated by using the $S(1,1)$ port of reflection coefficient $\&$ it was found to be $-14 \mathrm{~dB}$. In reference [15] the communication capabilities of short range millimeter wave in networkon-chip based on multi-core processors is investigated along with transmission capabilities between on-chip antennas for both inter and intra chip communication in multi-chip computing systems. The abilities of the wireless hubs existing in WiNoC to institute a postsilicon validation model for communication networks is discussed in [16]. A vertical top hat monopole positioned in Silicon Dioxide $\left(\mathrm{SiO}_{2}\right)$ as the wireless interconnect is presented in [17] and tried to achieve better radiation efficiency and impedance matching.
An implementation of a transmission channel for WiNoC applications is discussed considering integrated dipole antennas in $\mathrm{Ka}$ band using two antenna model in [18]. An effort is made to improvise the gain by shaping the chip packaging operating around $71 \mathrm{GHz}$ [19]. An extensive study of various aspects of chip scale wireless communications, including wave propagation, channel modelling, onchip Electromagnetics in millimeter wave and $\mathrm{THz}$ range is discussed in [20-23]. The influence of placement of the antenna and directionality on channel characteristics in the on-chip wireless channel analysis for multicore processors is discussed in [24] and analysis is extended to $\mathrm{THz}$ in [25]. Some of the related existing work for the analysis of on chip wireless link is summarized in Table 1. By and large, single and two antenna models have been generally examined from previous studies. The range of the frequencies varied between $20-70 \mathrm{GHz}$. We chose the 60-75 $\mathrm{GHz}$ band for the investigation since the frequency allotment for $60 \mathrm{GHz}$ wireless systems is becoming more popular. The focus of this research is on a four-antenna model for assessing the channel behaviour and receiving ability of three antennas when one of them is excited. In addition, we investigated the alignment and orientation of antenna positioning on a substrate using several antenna models. Based on the findings, we noticed that positioning the antenna in the edge center produces better results, which is discussed in this article. The results include a comparison of four antenna models in terms of receiving ability, as well as directivity and VSWR.

Table 1 Summary of associated existing works for analysis of the on-chip wireless link

\begin{tabular}{|c|c|c|c|}
\hline Reference & Observations & Frequency/ Antenna & Remarks \\
\hline [9] & $\begin{array}{l}\text { The effects of antenna placement and } \\
\text { directionality in the chip plane, as well as signal } \\
\text { loss, dispersion, and delay properties, are } \\
\text { discussed. }\end{array}$ & $\begin{array}{l}55-65 \mathrm{GHz}, \\
\text { folded dipole antenna and } \\
\text { planar log periodic antenna }\end{array}$ & $\begin{array}{l}\text { Analysis between pair of } \\
\text { antennas }\end{array}$ \\
\hline [15] & $\begin{array}{l}\text { Short-range } \mathrm{mm} \text { wave communication } \\
\text { capabilities among network-on-chip based multi- } \\
\text { core processors, integrated on a board were } \\
\text { investigated. }\end{array}$ & $\begin{array}{l}30 \mathrm{GHz}, \\
\text { zig-zag antenna }\end{array}$ & $\begin{array}{l}\text { The analysis is available only } \\
\text { with zig-zag antenna }\end{array}$ \\
\hline [18] & $\begin{array}{l}\text { Transmission channel analysis of WiNoC } \\
\text { applications }\end{array}$ & $\begin{array}{l}\text { Ka band }(26-40 \mathrm{GHz}) \text {, } \\
\text { Integrated dipole } \\
\text { antennas }\end{array}$ & $\begin{array}{l}\text { The analysis is only between } \\
\text { two antennas }\end{array}$ \\
\hline [22] & 3D models based on measurements in Ka band & $\begin{array}{l}\text { 20-50 GHz, } \\
\text { Dipole Antenna }\end{array}$ & $\begin{array}{l}\text { Highlights the performances } \\
\text { and trade-offs of different } \\
\text { parameters such as power } \\
\text { output and amplifier's gain }\end{array}$ \\
\hline [23] & $\begin{array}{l}\text { The implications on WiNoC design are presented } \\
\text { using a three-dimensional model that captures } \\
\text { essential chip components that influence wireless } \\
\text { transmission. }\end{array}$ & $\begin{array}{l}55-65 \quad \mathrm{GHz}, \quad \text { zig-zag, } \\
\text { planar log periodic antenna }\end{array}$ & $\begin{array}{l}\text { Analysis of individual and pair } \\
\text { of antennas }\end{array}$ \\
\hline
\end{tabular}




\section{Materials and methods}

\section{Construction and working of models}

Based on the investigation, triangular monopole, zigzag, meander and bowtie antennas were among the antenna models considered for analysis of intra-chip wireless communication and simulation using CST microwave Studio [11]. The steps for constructing the above-mentioned antennas are depicted in Figures 1 to 4 , respectively.

Single antenna models with chip dimensions of 1.75 $\mathrm{mm} \times 1.72 \mathrm{~mm} \times 0.49 \mathrm{~mm}$ [10] were considered and placed (various antennas) on it. The chip comprises two layers, with silicon used as a substrate in the bottom layer which is $0.36 \mathrm{~mm}$ thick and silicon dioxide used as a dielectric layer of thickness $0.12 \mathrm{~mm}$ with a thin copper plate as the ground plane at the bottom with the thickness of $0.01 \mathrm{~mm}$. Figure 1 depicts the overall block diagram.

Figure 2 to Figure 4 shows the detailed construction of a planar triangular monopole antenna [12] positioned on the chip's edge center, as well as the dimension and positioning of the antenna for simulation.

\section{Simulation of single antenna based on the considered parameters}

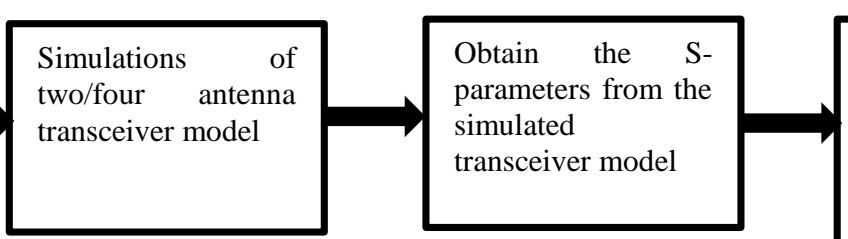

Analyse the Sparameters for the receptivity and losses to characterise the channel

Figure 1 Overall block diagram

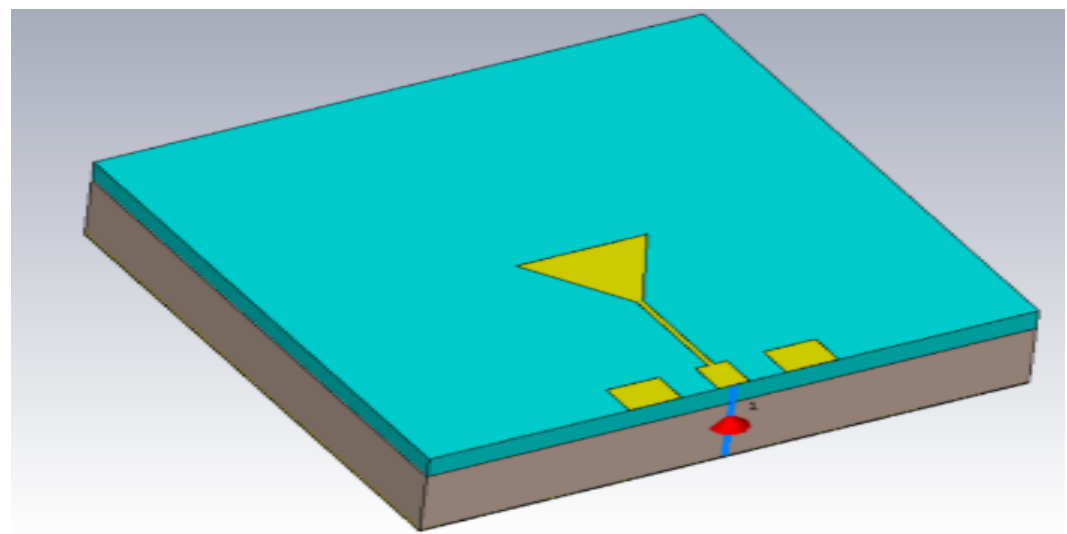

Figure 2 Single planar triangular monopole antenna

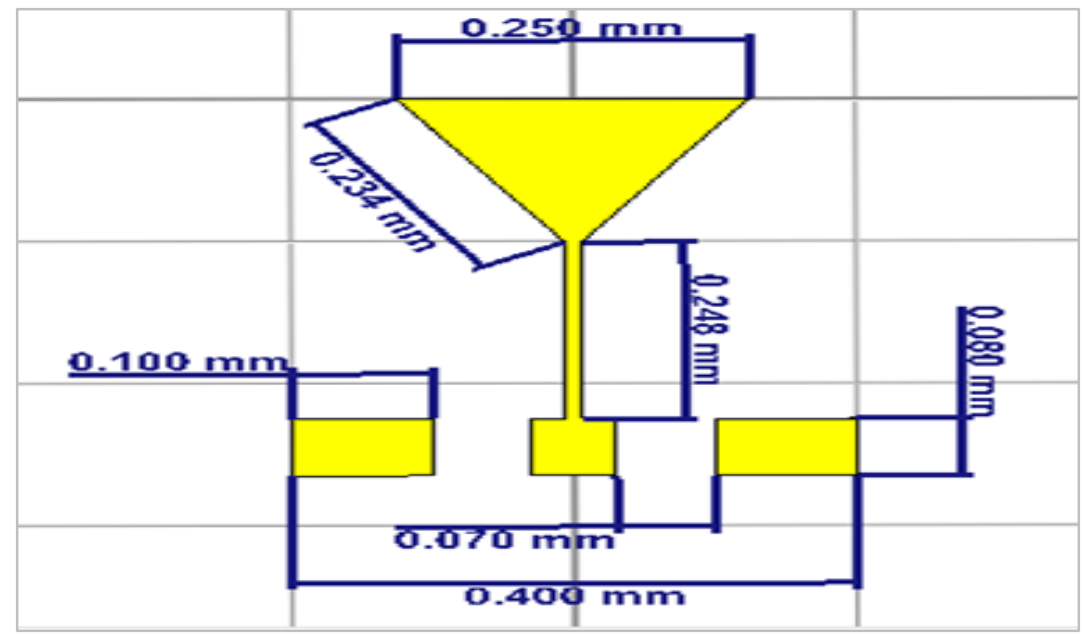

Figure 3 Dimensions of single planar triangular monopole antenna 


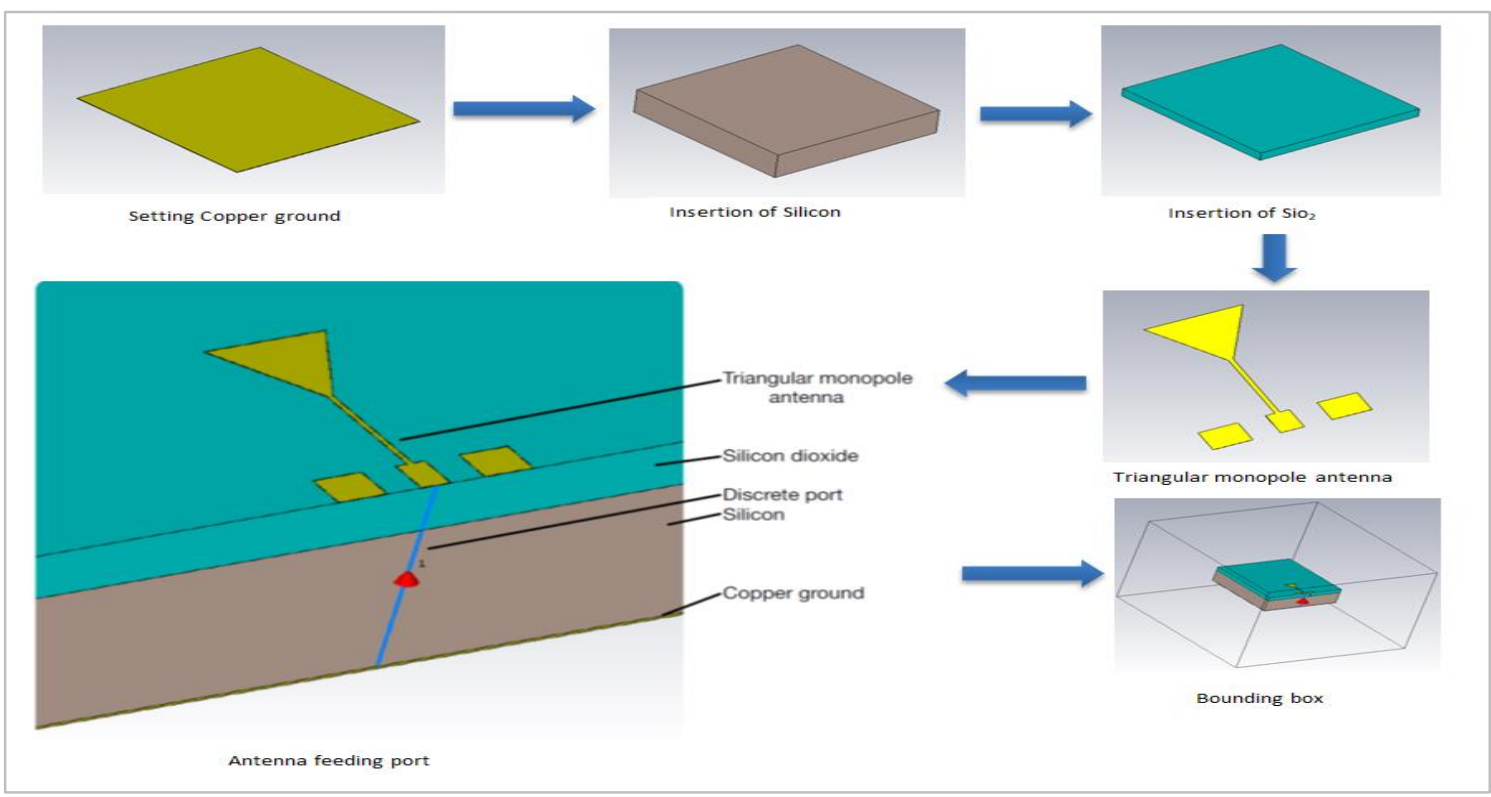

Figure 4 Construction of triangular monopole antenna

Similarly, the remaining three antennas were reconstructed as illustrated in Figure 4, using the dimensions of the zig-zag antenna [13], rectangular meander antenna, and the bowtie antenna [14] as shown in Figure 5 to Figure 7 . The rectangular meander antenna is designed by taking the total length of the antenna from the dipole calculation, then bending it into 24 arms of equal length and varying the antenna dimensions to achieve the desired results. The bowtie antenna is excited by applying a port to the extended arm.

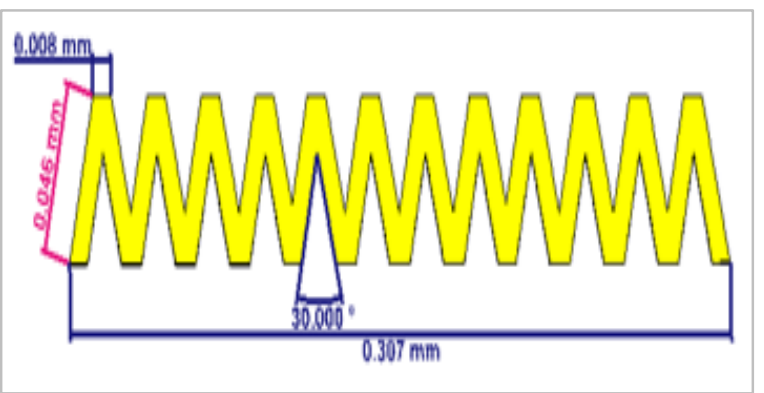

Figure 5 Dimensions of zig-zag antenna

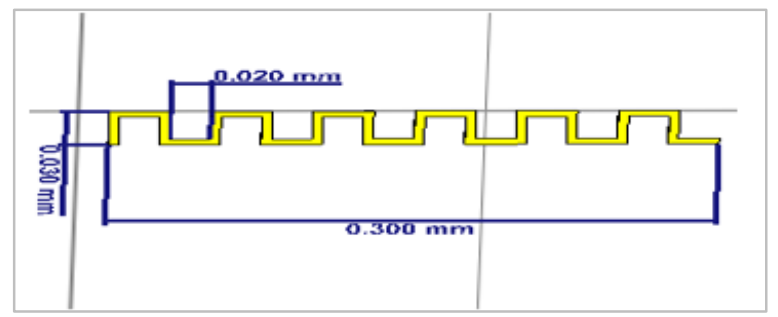

Figure 6 Dimensions of rectangular meander antenna 878

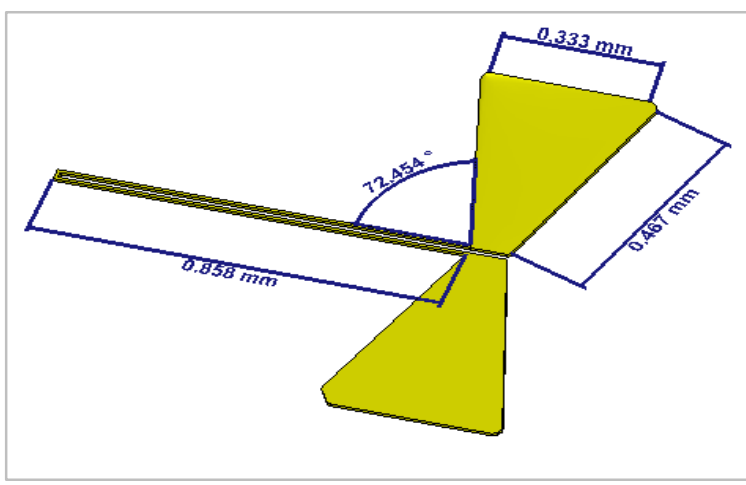

Figure 7 Dimensions of bowtie antenna

\section{Results and discussions}

The Gaussian signal shown in Figure 8 was used as the input signal for all of the models in this research to analyze the models over a range of frequencies. This signal is applied to the discrete port which has $50 \mathrm{ohms}$ matched impedance.

The results of all four single antenna were compared and presented in Table 2. It is indicated from the results that the bowtie antenna gives the best result with a S11 value of $-46.02 \mathrm{~dB}$ and a VSWR value of 1.01. The resonant frequency of the single antenna model simulated is close to $75 \mathrm{GHz}$. The single antenna models were used to create four on-chip antenna transceiver system models. For on-chip antenna, the antenna is kept in far-field as this boundary is considered at a distance of $(\lambda / 2 \pi)$ from the antenna. 


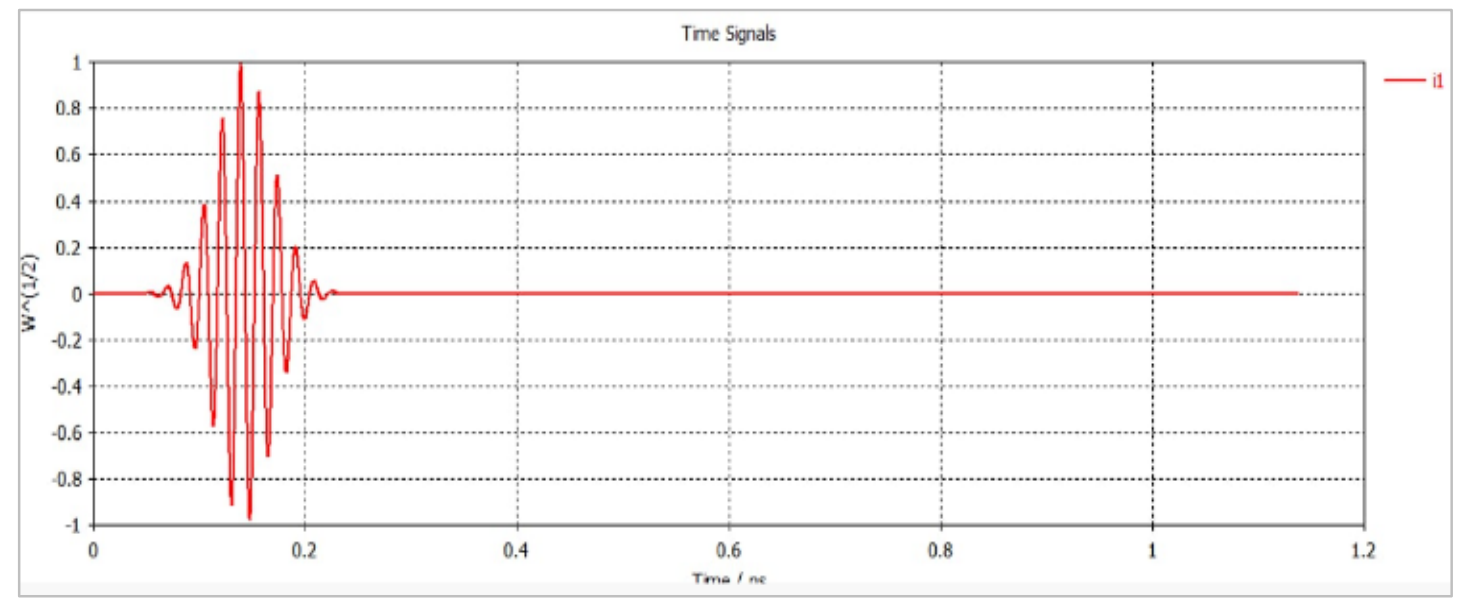

Figure 8 Gaussian input signal

Table 2 Results for various single antenna models

\begin{tabular}{llll}
\hline Antenna used & Resonant frequency $(\mathbf{G H z})$ & $\mathbf{S}_{\mathbf{1 1}}(\mathbf{d B})$ & VSWR \\
\hline Triangular Monopole & 75.08 & -6.81 & 2.68 \\
\hline Rectangular Meander & 75.95 & -17.19 & 1.32 \\
\hline Bowtie & 74.04 & -46.02 & 1.01 \\
\hline Zig-Zag & 75.56 & -17.05 & 1.33 \\
\hline
\end{tabular}

4.1Construction of four antenna Transceiver system model using a bowtie antenna

The construction and description of bowtie four antenna transceiver system is depicted in Figure 9. The dimensions of the chip used for four antenna model were $5 \mathrm{~mm} \times 5 \mathrm{~mm} \times 0.633 \mathrm{~mm}$ as illustrated in Figure 10. Here port 1 serves as the source port which acts as a transmitter and the rest of the ports act as receivers. Silicon is used as substrate of $0.633 \mathrm{~mm}$ thickness. At top $\mathrm{SiO}_{2}$ layer is placed with a thickness of $0.002 \mathrm{~mm}$. A copper plate of $0.001 \mathrm{~mm}$ thickness is used as ground plane reference to ports.

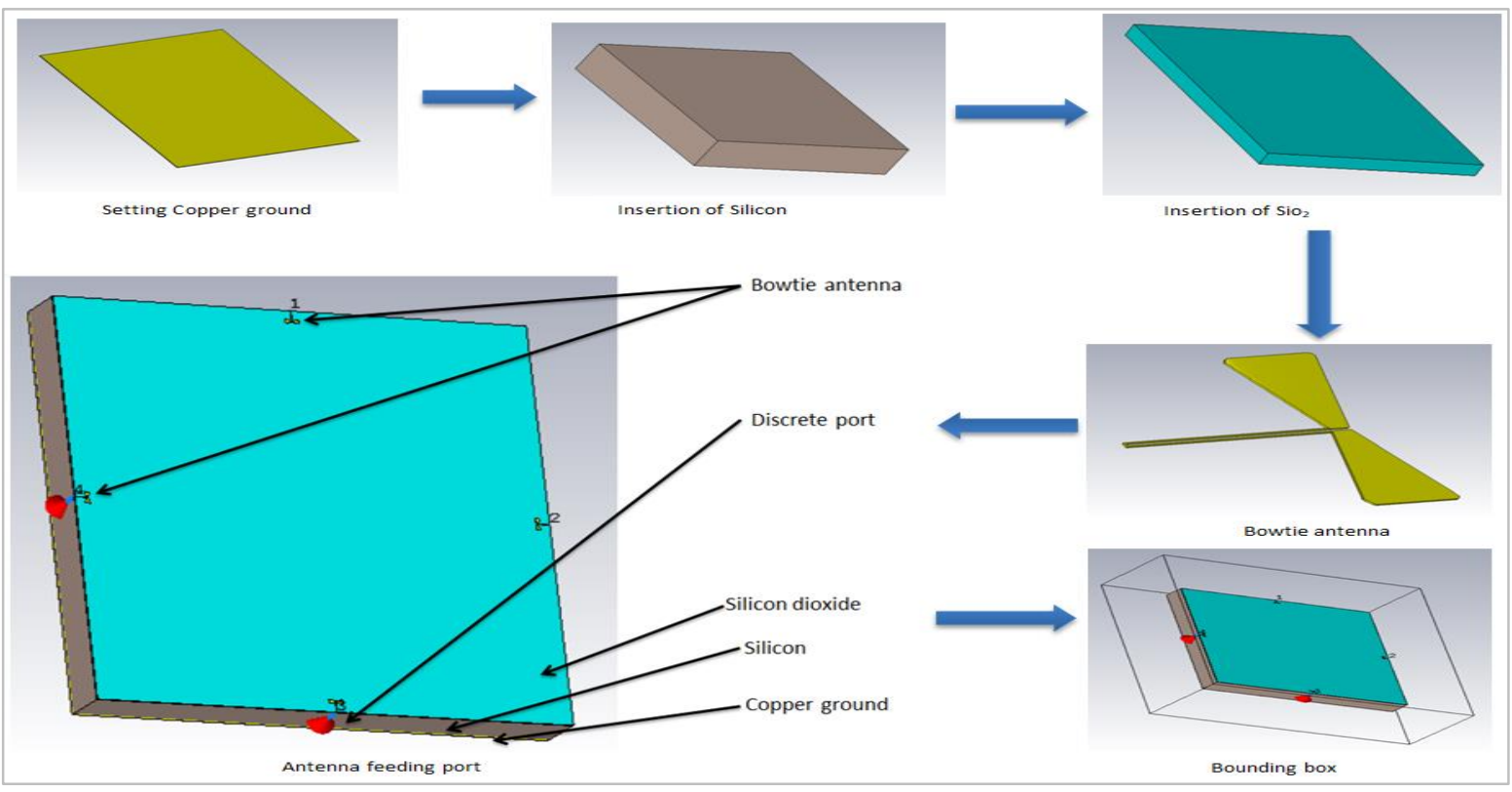

Figure 9 Construction of four antenna transceiver system 


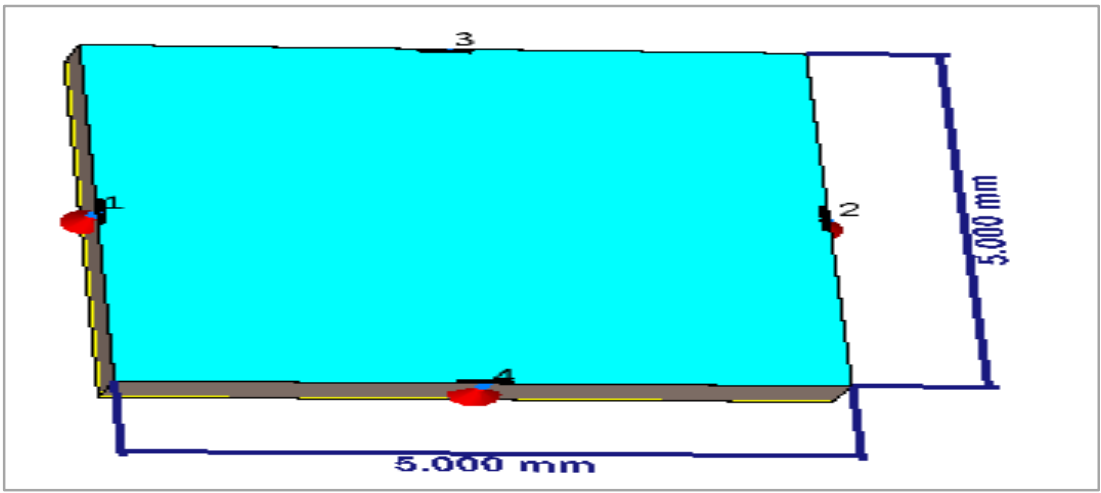

Figure 10 Dimensions of chip used for four antenna system

All of the antennas were positioned at the edge center of the model and their dimensions are identical to those of a single antenna system. Figure 11 depicts the directivity of a bowtie antenna, which shows a more directive beam towards antenna-2 and antenna-4 than antenna-3. Figures 12 and 13 depict the $\mathrm{S}$ parameters as well as the VSWR. Here port-1 is exited while all other ports are receiving. The value of $\mathrm{S} 11$ is -17.22 $\mathrm{dB}$ at $65.18 \mathrm{GHz}$. S21, S31, S41 shows the receptivity of other antennas when antenna-1 is transmitting.

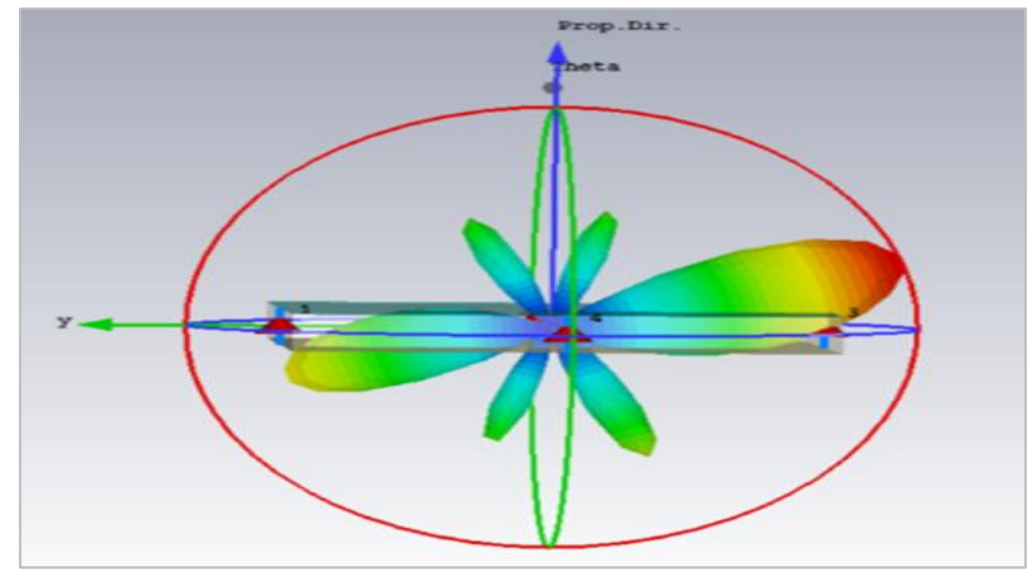

Figure 11 Directivity of bowtie antenna used in four antenna system

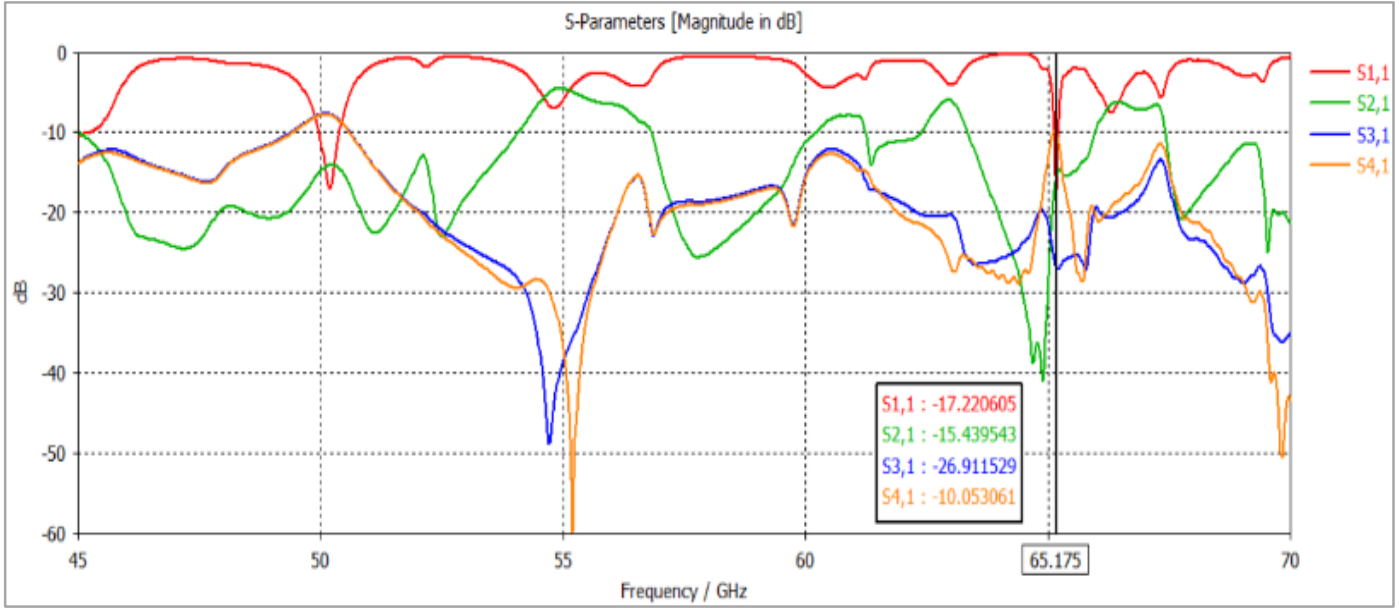

Figure $12 \mathrm{~S}$ parameters of a bowtie antenna used in four antenna system 880 


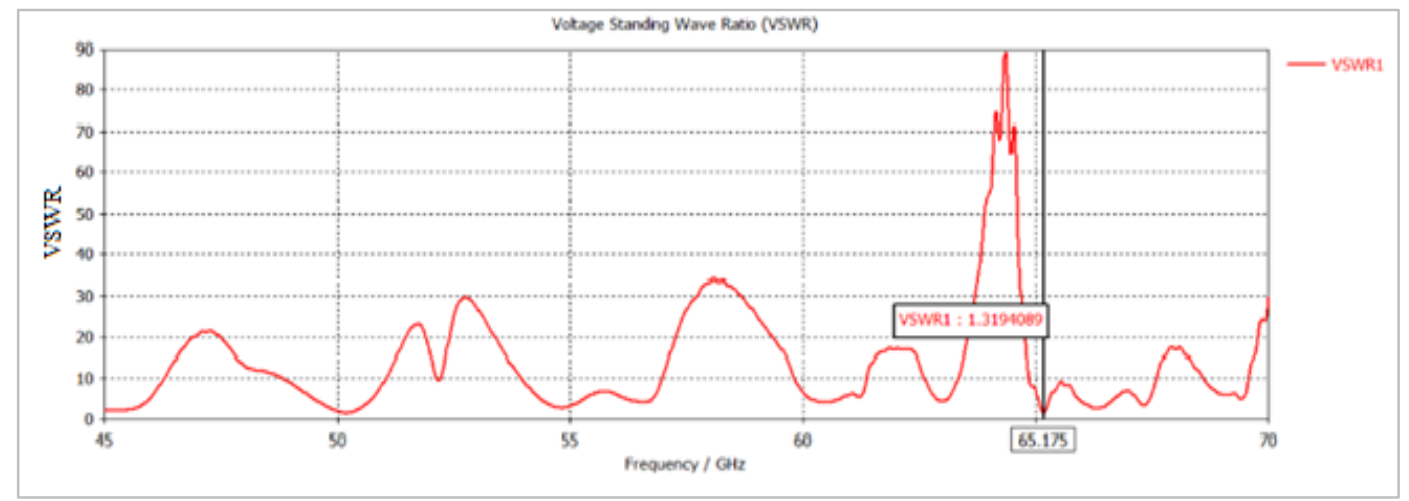

Figure 13 VSWR of bowtie antenna used in four antenna system

\subsection{Planar triangular monopole transceiver system} with four antennas

The placement and directivity of planar triangular monopole are shown in Figure 14 and Figure 15. All the antennas are located on the chip's edge center and their dimensions are the same as in a single antenna system. The $\mathrm{S}$ parameters are shown in Figure 16 and VSWR in Figure 17. VSWR for this configuration is around 2 which is not so good compare to other

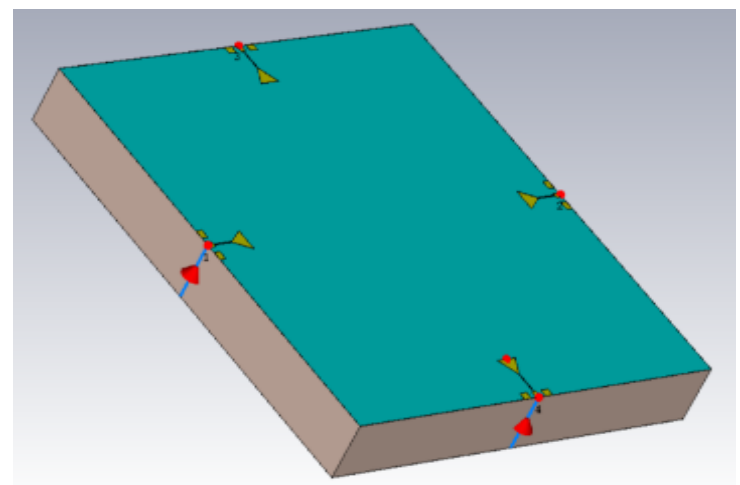

Figure 14 Placement of triangular monopole antenna system antenna models. As depicted in Figure 15, the receptivity of all three antennas which are placed at sides and opposite to source port is approximately equal. It does not radiate backwards, which is a major benefit. When considering the S-Parameters, the S11 is not ideal, but the receptivity of other antennas placed to the sides and opposite of the radiating antenna is good.

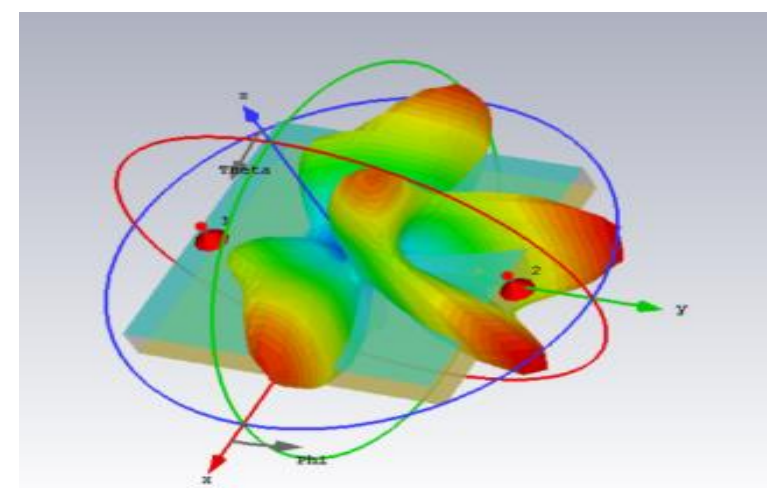

Figure 15 Directivity of triangular monopole antenna in four antenna in four antenna system

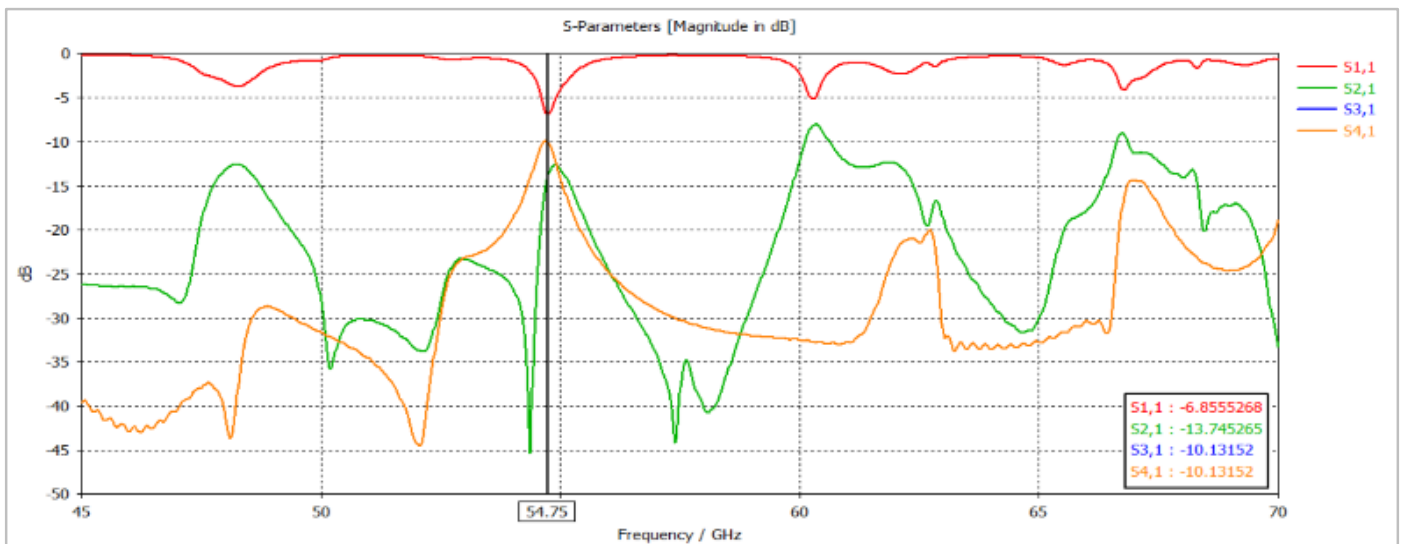

Figure $16 \mathrm{~S}$ parameters of triangular monopole antenna used in four antenna system 


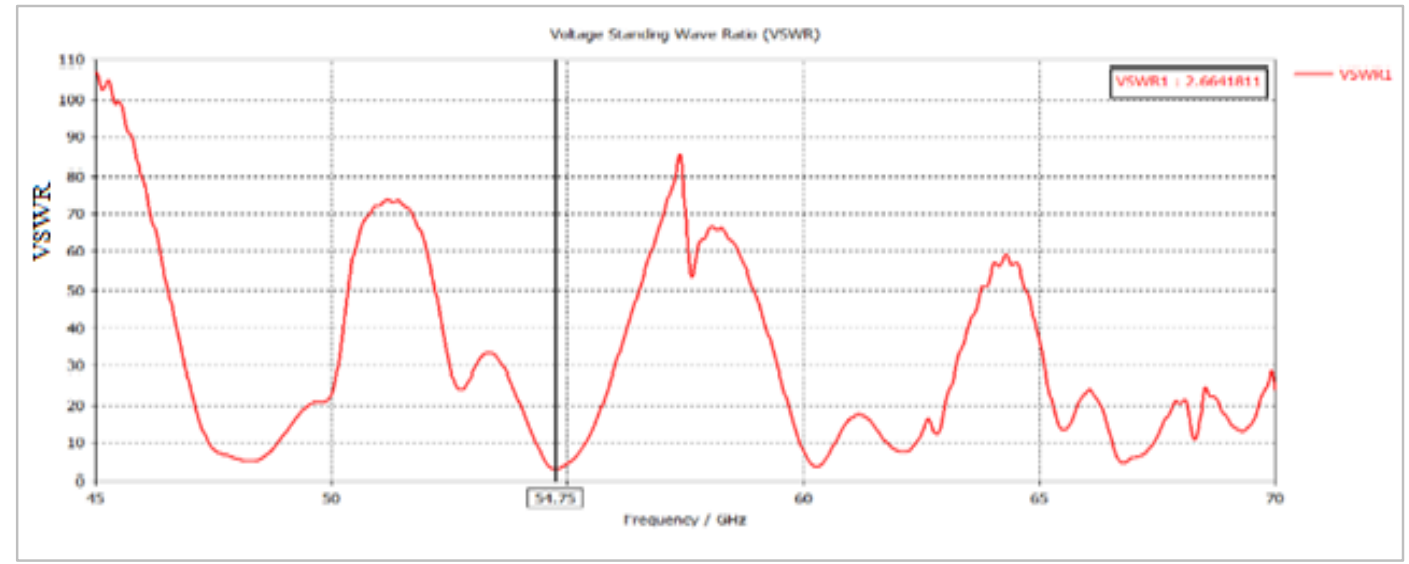

Figure 17 VSWR of triangular monopole antenna used in four antenna system

4.3Zig-zag transceiver system with four antennas

The placement of four zig-zag antennas on the chip's edge center is shown in Figure 18. The antenna's dimensions are identical to those of a single antenna system. Figure 19 depicts the $\mathrm{S}$ parameter analysis. $\mathrm{S} 11$ shows the return loss which is $-10.31 \mathrm{~dB}$ at 54.9 $\mathrm{GHz}$. When Port 1 is excited, S21, S31, and S41 show the receptivity of other antennas. Antennas-3 and 4 have superior results since they are located on the sides, however, antenna-2 has less receptivity because it is placed on the other side, as illustrated in Figure 20. At $54.9 \mathrm{GHz}$, the VSWR is 1.88 , which is acceptable.

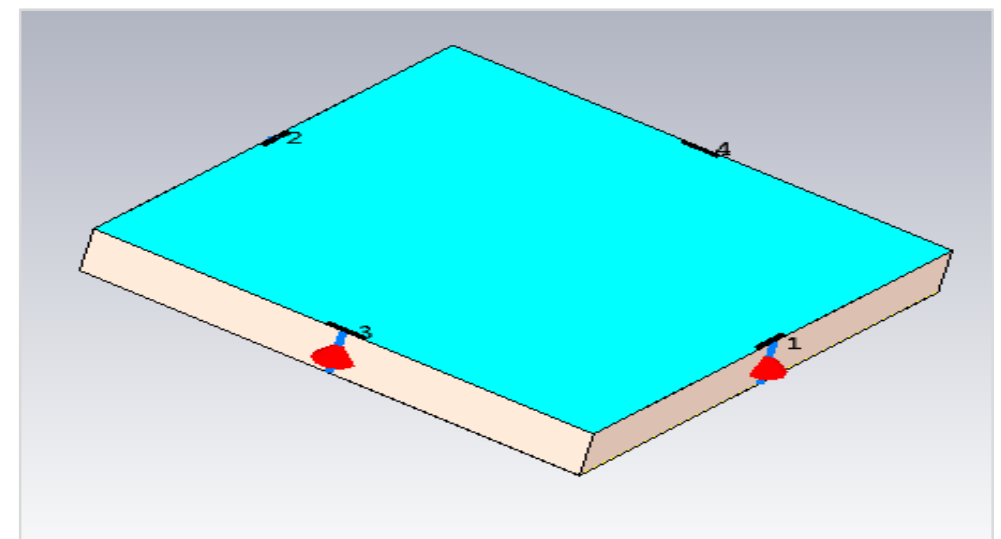

Figure 18 Placement of zig-zag antennas used in four antenna system

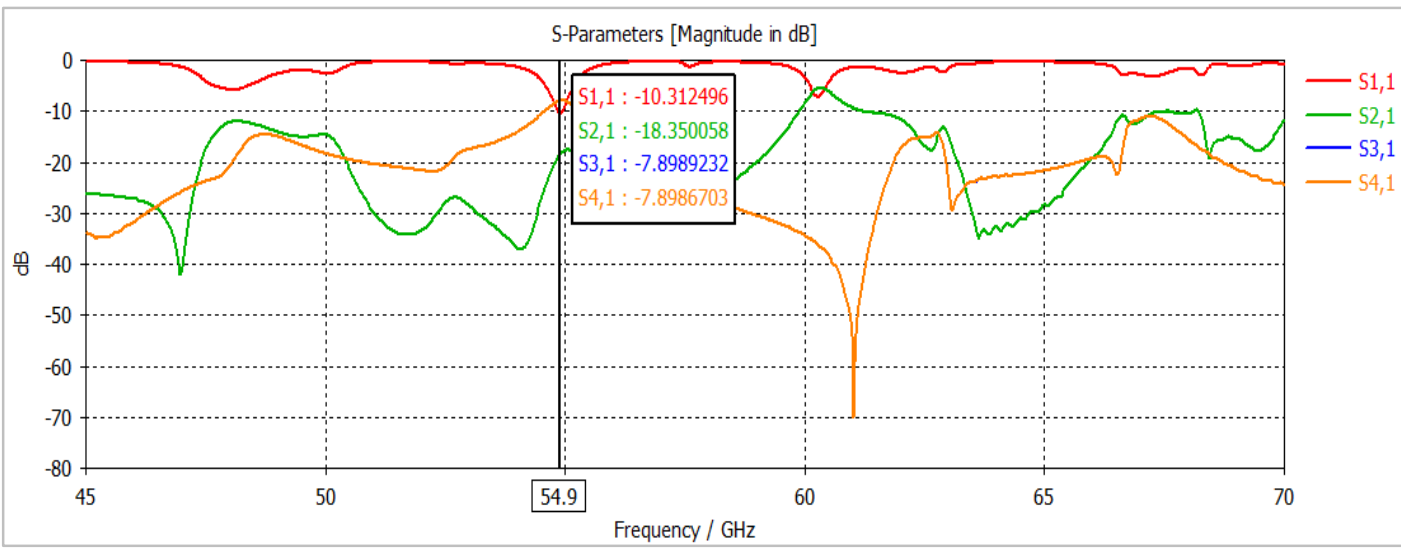

Figure $19 \mathrm{~S}$ parameters of zig-zag antennas used in four antenna system 


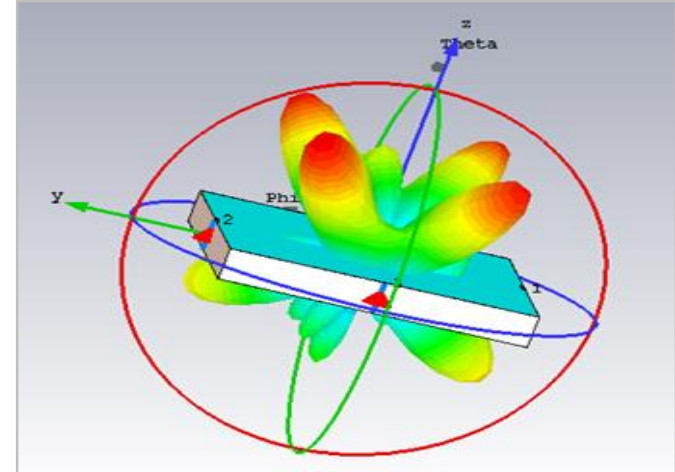

Figure 20 Directivity of zig-zag antenna used in four antenna system

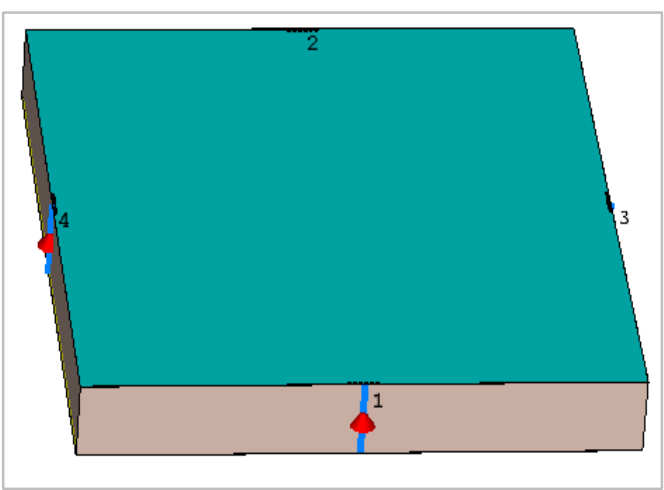

Figure 21 Placement of rectangular meander antenna in 4 antenna system

\subsection{Rectangular meander transceiver system with} four antennas

Figure 21 depicts the positioning of four antennas on the chip's edge center, while Figure 22 depicts their directivity. All antenna dimensions are same as in a single antenna system. In the analysis of S parameters presented in Figure 23, the $\mathrm{S} 11$ at $55.1 \mathrm{GHz}$ is approximately $-15.81 \mathrm{~dB}$, which is within acceptable limits. The receptivity of three antennas can be seen in the directivity plot, where the radiation is directed more to the sides than the opposite as indicated by major lobes. The VSWR is 1.39 , which is considered to be satisfactory.

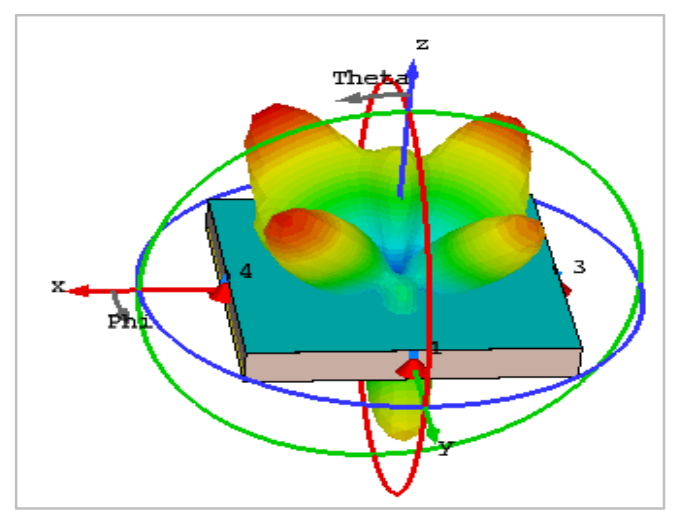

Figure 22 Directivity of rectangular meander antenna used used in four antenna system

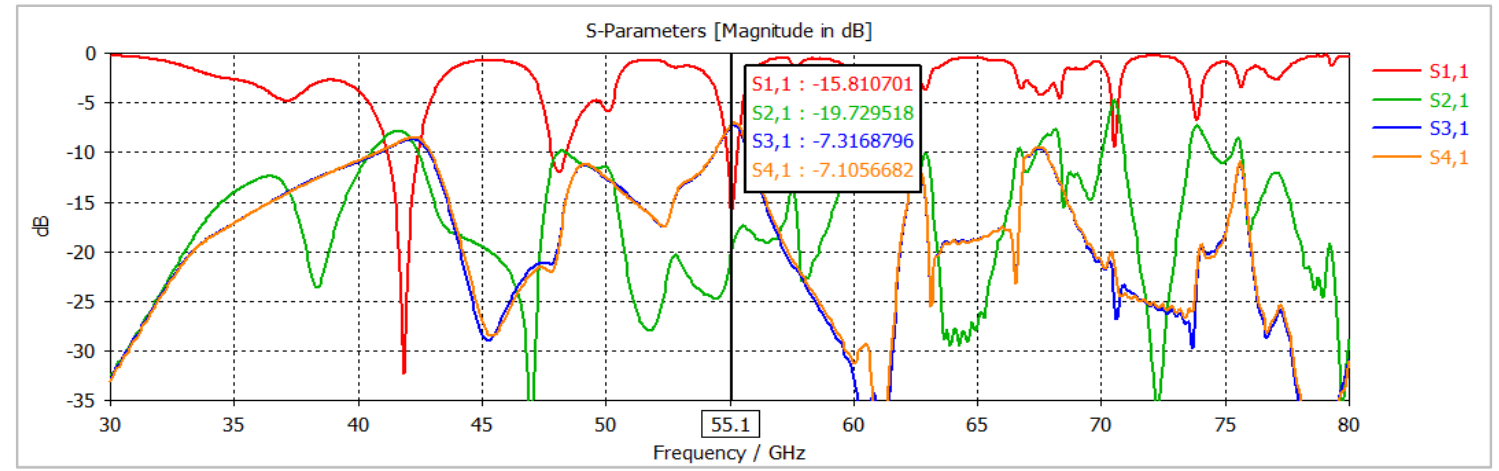

Figure $23 \mathrm{~S}$ parameters of the rectangular meander antenna used in 4 antenna system

Table 3 shows the results for four different antenna models with antennas located on the edge centre. The bowtie antenna has a better reflection coefficient S11 at $-17.22 \mathrm{~dB}$. The receptivity of the antenna 2 and 4 are marginally better, but very less receptivity shown by antenna 3. VSWR 1.32 is satisfactory. In the case of a zig-zag antenna, the value obtained for S11 at $10.31 \mathrm{~dB}$ is reasonable. It is observed that the sidelobes have more directivity, resulting in transmission coefficients $\mathrm{S} 31$ at $-7.9 \mathrm{~dB}, \mathrm{~S} 41$ at -7.9 $\mathrm{dB}$ of antennas- 3 and 4 on the sideways being significantly higher than $\mathrm{S} 21$ at $-18.35 \mathrm{~dB}$ of antenna 2 on the opposite side of the source port. The obtained resonance frequency is $54.90 \mathrm{GHz}$. VSWR of 1.88 is also satisfactory. The results obtained for the rectangular meander antenna are similar to that of zigzag antenna configuration with slightly better reflection loss $\mathrm{S} 11$ at $-15.81 \mathrm{~dB}$ and VSWR with 1.39. However, the main lobe directivity S21 at $-19.73 \mathrm{~dB}$ is marginally less than zig-zag configuration. The transmission coefficients $\mathrm{S} 31$ at $-7.32 \mathrm{~dB}, \mathrm{~S} 41$ at -7.11 $\mathrm{dB}$ of antenna 3 and 4 placed in the sideways has better 
receptivity compared to the antenna placed opposite to the source port. The obtained resonant frequency is $55.10 \mathrm{GHz}$. The performance of the triangular monopole is the least of all considering the S11 at $6.85 \mathrm{~dB}$, which is less than the required value. However the directivity of main and sidelobes are quite satisfactory which results in good receptivity of transmitted signal to the opposite and sideways antenna. It is observed that the resonant frequency for this configuration is $54.75 \mathrm{GHz}$. Table 4 compares this study to earlier work on zig-zag antenna models. The achieved findings are respectable when compared to the overall response of the simulated models. Complete list of abbreviations is shown in Appendix I.

\section{Limitations of the Study}

In this work, analysis is carried out under ideal conditions. However, the actual scenario of intra chip wireless communication includes the effect of other metal interconnects. The influence of various materials on wireless communication in intra chip is also an important issue that can be taken up by the research community.

Table 3 Results of various four antenna models with antennas on edge center

\begin{tabular}{lllllll}
\hline Antenna & $\begin{array}{l}\text { Resonant } \\
\text { Frequency }(\mathbf{G H z})\end{array}$ & $\mathbf{S 1 1}(\mathbf{d B})$ & $\mathbf{S 2 1}(\mathbf{d B})$ & $\mathbf{S 3 1}(\mathbf{d B})$ & S41 (dB) & VSWR \\
\hline Bowtie & 65.18 & -17.22 & -15.44 & -26.91 & -10.05 & 1.32 \\
\hline Triangular Monopole & 54.75 & -6.85 & -13.74 & -10.13 & -10.13 & 2.66 \\
\hline Zig-Zag & 54.90 & -10.31 & -18.35 & -7.90 & -7.90 & 1.88 \\
\hline Rectangular Meander & 55.1 & -15.81 & -19.73 & -7.32 & -7.11 & 1.39 \\
\hline
\end{tabular}

Table 4 Comparison with previous work

\begin{tabular}{llll}
\hline Reference[15] & & \multicolumn{2}{c}{ Present work } \\
\hline Parameters & Simulated & Measured & Simulated \\
\hline Frequency & $30-32 \mathrm{GHz}$ & $20 \mathrm{GHz}$ to $30 \mathrm{GHz}$ & $50-70 \mathrm{GHz}$ \\
\hline $\begin{array}{llll}\text { Transmission } \\
\text { Coefficients }\end{array}$ & $\begin{array}{l}\text {-31 dB to }-38 \\
\text { dB }\end{array}$ & $-35 \mathrm{~dB}$ to $-60 \mathrm{~dB}$ & $-7.90 \mathrm{~dB}$ to $-18 \mathrm{~dB}$ \\
\hline Zig-zag Antenna & 4 & - & 4 \\
\hline Distance & $10 \mathrm{~mm}$ & $10 \mathrm{~mm}$ & $5 \mathrm{~mm}$ \\
\hline Simulating Tool & ANSYS HFSS & Hardware & CST \\
\hline
\end{tabular}

\section{Conclusions and future scope}

Four on-chip antenna geometries are analyzed in multi-antenna models and their results are compared. In case of the single antenna model, bowtie antenna is found to provide better performance in terms of efficiency and reflection coefficient. Meander antenna has the better values of reflection coefficient and transmission coefficient for receiving antenna 2 placed opposite to transmitting antenna among all the four antenna models tested. In the zig-zag four antenna model, the transmission coefficient for receiving antennas-3 and 4 placed on adjacent sides of the transmitting antenna is significantly higher than that of the antenna placed opposite to the source port. If VSWR and S11 are critical then the use of triangular monopole is not recommended.
The future scope of the work includes the performance analyses of different antenna geometries and antenna placements. To obtain even better outcomes, the results from CST Microwave Studio can be integrated into MATLAB Simulink. Further analysis can be carried out with other metals like silver and gold instead of copper. Various other semiconductor substrate materials such as Graphene, InAs, etc. can also be tried.

\section{Acknowledgment}

We are very thankful to Ayush Prakash, Sandeep Kumar Shrey and Amarendra for helping in building antenna models.

\section{Conflicts of interest}

The authors have no conflicts of interest to declare. 


\section{References}

[1] Abadal S, Han C, Jornet JM. Wave propagation and channel modeling in chip-scale wireless communications: a survey from millimeter-wave to terahertz and optics. IEEE Access. 2019; 8:278-93.

[2] Neculoiu D, Muller A, Tang K, Laskin E, Voinigescu SP. $160 \mathrm{GHz}$ on-chip dipole antenna structure in silicon technology. In international semiconductor conference 2007 (pp. 245-8). IEEE.

[3] Tao C, Teemu P, Esa T, Tenhunen H. RF transceiver circuit technology based wireless interconnects for inter-and intra-chip communication system. In electronics packaging technology conference 2008 (pp. 1409-14). IEEE.

[4] Chang K, Deb S, Ganguly A, Yu X, Sah SP, Pande PP, et al. Performance evaluation and design trade-offs for wireless network-on-chip architectures. ACM Journal on Emerging Technologies in Computing Systems. 2012; 8(3):1-25.

[5] Barakat A, Allam A, Pokharel RK, Elsadek H, El-sayed M, Yoshida K. $60 \mathrm{Ghz}$ triangular monopole antennaon-chip over an artificial magnetic conductor. In European conference on antennas and propagation 2012 (pp. 972-6). IEEE.

[6] Samaiyar A, Ram SS, Deb S. Millimeter-wave planar $\log$ periodic antenna for on-chip wireless interconnects. In the European conference on antennas and propagation 2014 (pp. 1007-9). IEEE.

[7] Wang W, Chen Y, Yang S, Cao Q, Li H, Zheng X, et al. Wireless inter/intra-chip communication using an innovative PCB channel bounded by a metamaterial absorber. IEEE Antennas and Wireless Propagation Letters. 2016; 15:1634-7.

[8] Pizano-escalante L, Longoria-gandara O, Parra-michel R, Peña-campos F. Simulation model to predict BER based on s-parameters of high-speed interconnects. IEEE Design \& Test. 2018; 36(1):31-9.

[9] Gade SH, Rout SS, Deb S. On-chip wireless channel propagation: impact of antenna directionality and placement on channel performance. In international symposium on networks-on-chip 2018 (pp. 1-8). IEEE.

[10] Elaiyabharathi N, Muthukannan P, Reji M. Design and simulation of meander line antenna for wireless applications. International Journal of Recent Technology and Engineering. 2019; 8(1):2001-5.

[11] https://www.3ds.com/productsservices/simulia/products/cst-studio-suite/. Accessed 10 May 2021.

[12] Upadhyay S, Srivastava S. A $60-\mathrm{GHz}$ on-chip monopole antenna using silicon technology. In applied electromagnetics conference 2013 (pp. 1-2). IEEE.

[13] Deb S. Millimeter-wave wireless network-on-chip: a CMOS compatible interconnection infrastructure for future many-core processors. Washington State University; 2012.
[14] Karamzadeh S, Kiliç OF, Hepbiçer AS, Demirbaş F. Bow tie antenna design for GPR applications. International Journal of Electronics Mechanical and Mechatronics Engineering. 2016; 6(2):1187-94.

[15] Narde RS, Venkataraman J, Ganguly A, Puchades I. Intra-and inter-chip transmission of millimeter-wave interconnects in NoC-based multi-chip systems. IEEE Access. 2019; 7:112200-15.

[16] Rout SS, Basu K, Deb S. Efficient post-silicon validation of network-on-chip using wireless links. In 32nd international conference on VLSI design and international conference on embedded systems 2019 (pp. 371-6). IEEE.

[17] Narde RS, Venkataraman J, Ganguly A. Disc-loaded, vertical top-hat monopole antenna at $225 \mathrm{GHz}$ for onchip wireless communications. In international symposium on antennas and propagation and USNCURSI radio science meeting 2019 (pp. 1883-4). IEEE.

[18] El MI, Le GT, Martin PM, Allanic R, Quendo C. Integrated dipole antennas and propagation channel on silicon in $\mathrm{Ka}$ band for $\mathrm{WiNoC}$ applications. In workshop on signal and power integrity 2018 (pp. 1-4). IEEE.

[19] Zhang H, Shamim A. Gain enhancement of millimeterwave on-chip antenna through an additively manufactured functional package. IEEE Transactions on Antennas and Propagation. 2020; 68(6):4344-53.

[20] El MI, Le GT, Martin PM, Allanic R, Quendo C. Electromagnetic characterization of the intrachip propagation channel in \$ Ka \$-and \$ V \$-bands. IEEE Transactions on Components, Packaging and Manufacturing Technology. 2019; 9(10):1931-41.

[21] Timoneda X, Cabellos-aparicio A, Manessis D, Alarcón E, Abadal S. Channel characterization for chip-scale wireless communications within computing packages. In international symposium on networks-onchip 2018 (pp. 1-8). IEEE.

[22] El MI, Martin PM, Mondal HK, Allanic R, Le GT, Quendo C, et al. Accurate channel models for realistic design space exploration of future wireless NoCs. In international symposium on networks-on-chip 2018 (pp. 1-8). IEEE.

[23] Gade SH, Ram SS, Deb S. Millimeter wave wireless interconnects in deep submicron chips: challenges and opportunities. Integration. 2019; 64:127-36.

[24] Timoneda X, Abadal S, Franques A, Manessis D, Zhou $\mathrm{J}$, Torrellas J, et al. Engineer the channel and adapt to it: enabling wireless intra-chip communication. IEEE Transactions on Communications. 2020; 68(5):324758.

[25] Chen Y, Han C. Channel modeling and analysis for wireless networks-on-chip communications in the millimeter wave and terahertz bands. In INFOCOM conference on computer communications workshops 2018 (pp. 651-6). IEEE. 


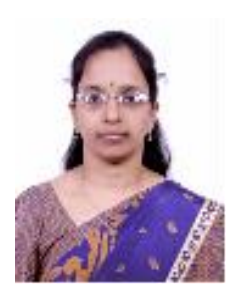

Deepa N P, obtained her Bachelor's degree in Electronics and Communication Engineering from Kuvempu University, Karnataka, India and Masters in Digital Communication Engineering from Visvesvaraya Technological University, Belagavi, Karnataka, India. She is a part time research scholar in the field of short range wireless communication and has 15 years of teaching experience in various engineering colleges. She has guided many postgraduate and undergraduate students for their final year projects. She has published research papers in various journals and national / international conferences. Her area of interest includes Wireless communication, Network on Chip, RF and Microwave, Signal Processing and Image processing.

Email: deepnp@gmail.com

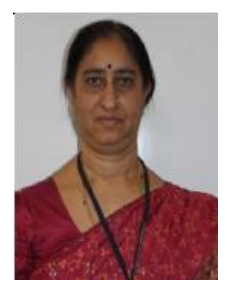

Dr. K. L. Sudha, obtained her Bachelor's degree in Electronics and Communication engineering from Mysore University and Masters from Bangalore University. She got Ph.D for her work on "Detection of FH CDMA signals in time varying channel" from Osmania University, Hyderabad. She has 20 years of teaching experience in Engineering Colleges. She has executed two ISRO funded research projects successfully as principal investigator. She is supervising seven $\mathrm{PhD}$ scholars and has guided many postgraduate and undergraduate students for their projects. She has published more than 60 research papers in national / International journals and conferences. Her research interests are Wireless Communication, Coding Theory, Image Processing and Chaotic Theory.

Email: drsudha-ece@dayanandasagar.edu

\begin{tabular}{|c|c|c|}
\hline \multicolumn{3}{|c|}{ Appendix I } \\
\hline S.No. & Abbreviation & Description \\
\hline 1 & $\mathrm{AC}$ & Alternating Current \\
\hline 2 & $\mathrm{AOC}$ & Antenna-On-Chip \\
\hline 3 & AMC & Artificial Magnetic Conductor \\
\hline 4 & BER & Bit Error Rate \\
\hline 5 & CMOS & $\begin{array}{lll}\text { Complementary } & \text { Metal } & \text { Oxide } \\
\text { Semiconductor } & & \end{array}$ \\
\hline 6 & HFSS & High-Frequency Structure Simulator \\
\hline 7 & HIS & High-Speed Interconnect \\
\hline 8 & IC & Integrated Circuits \\
\hline 9 & PCB & Printed Circuit Board \\
\hline 10 & PLPA & Planar Log-Periodic Antenna \\
\hline 11 & RF & Radio Frequency \\
\hline 12 & RFWI & $\begin{array}{l}\text { Radio Frequency } \\
\text { Interconnect }\end{array}$ \\
\hline 13 & S11 & Return Loss \\
\hline 14 & $\mathrm{SiO}_{2}$ & Silicon Dioxide \\
\hline 15 & SoC & System On Chip \\
\hline 16 & TSV & Through-Silicon-Via \\
\hline 17 & VLSI & Very Large Scale Integration \\
\hline 18 & VSWR & Voltage Standing Wave Ratio \\
\hline 19 & WIIC & Wireless Inter/Intra-Chip \\
\hline 20 & WiNoC & Wireless-Networks-on-Chip \\
\hline 21 & WiMAX & $\begin{array}{l}\text { Worldwide Interoperability for } \\
\text { Microwave Access }\end{array}$ \\
\hline
\end{tabular}

